\title{
Erratum: Wess-Zumino and super Yang-Mills theories in $D=4$ integral superspace
}

\author{
L. Castellani, ${ }^{a, b, c}$ R. Catenacci ${ }^{a, c, d}$ and P.A. Grassi ${ }^{a, b, c, e}$ \\ a Dipartimento di Scienze e Innovazione Tecnologica, Università del Piemonte Orientale, \\ viale T. Michel 11, 15121 Alessandria, Italy \\ ${ }^{b}$ INFN, Sezione di Torino, \\ via P. Giuria 1, 10125 Torino, Italy \\ ${ }^{c}$ Arnold-Regge Center, \\ via P. Giuria 1, 10125 Torino, Italy \\ ${ }^{d}$ Gruppo Nazionale di Fisica Matematica, INdAM, \\ p.le Aldo Moro 5, 00185 Roma, Italy \\ e Yukawa Institute for Theoretical Physics, Kyoto University, \\ Kitashirakawa Oiwakecho, Sakyo Ward, Kyoto 606-8317, Japan \\ E-mail: leonardo.castellani@uniupo.it, roberto.catenacci@uniupo.it, \\ pietro.grassi@uniupo.it
}

ERratum to: JHEP05(2018)040

ARXIV EPRINT: 1711.07194

\section{Acknowledgments}

We thank P. Fré and C. Maccaferri for fruitful discussions. This research is original and has a financial support of the Università del Piemonte Orientale (Fondi Ricerca Locale).

Open Access. This article is distributed under the terms of the Creative Commons Attribution License (CC-BY 4.0), which permits any use, distribution and reproduction in any medium, provided the original author(s) and source are credited. 